CARDIOLOGY

\title{
Hybrid Coronary Revascularization and the Hybrid Cardiovascular Operating Room
}

\author{
Konstantinos Dean Boudoulas ${ }^{a}$ Konstantinos Marmagkiolis ${ }^{b}$ \\ Antonios A. Pitsis ${ }^{c}$ Harisios Boudoulas ${ }^{a, d}$ \\ ${ }^{a}$ Division of Cardiovascular Medicine, Department of Medicine, The Ohio State University, Columbus, OH, USA; \\ ${ }^{b}$ Florida Hospital, Pepin Heart Institute, Tampa, FL, USA; ' $A$ Agios Lukas Hospital, Thessaloniki, Greece; \\ ${ }^{\mathrm{d} B i o m e d i c a l}$ Research Foundation, Academy of Athens, Athens, Greece
}

Currently, two major techniques are used for coronary artery revascularization: traditional coronary artery bypass grafting $(\mathrm{CABG})$ surgery and percutaneous coronary intervention (PCI) with angioplasty and/or stent placement. Each approach has its advantages and limitations, and one method is considered complimentary to the other $[1,2]$. PCI is less invasive and it is associated with a lower procedural morbidity and mortality, but the use of dual antiplatelet therapy required after stent placement may constitute a problem, at least in certain patients. Further, PCI yields suboptimal results in patients with diabetes mellitus and chronic kidney disease, and in saphenous vein grafts. Moreover, in patients with multiple coronary artery stenoses, complete revascularization cannot always be achieved with PCI. CABG is more invasive and it is associated with a higher morbidity and mortality including periprocedural stroke, as compared to PCI; however, CABG more often provides complete revascularization in patients with multivessel coronary artery disease that is associated with better long-term outcomes including a decrease in repeat revascularization, as compared to PCI. In addition, use of the internal mam-
๑) 2018 S. Karger AG, Basel mary (thoracic) artery anastomosis to the left anterior descending (LAD) artery gives excellent long-term results; this may be the single most important factor in the superior survival benefit seen with CABG compared to PCI. In certain patients, a hybrid approach (i.e., left internal mammary artery anastomosis to the LAD and PCI to the remaining arteries) can be used $[1,3]$. Left internal mammary artery anastomosis to the LAD can be performed off-pump with a minimally invasive surgical procedure with fewer procedural risks while utilizing the LIMA, which is associated with excellent long-term patency and with a survival benefit. In addition, advancements in stent technology and medical management have resulted in a reduction in the rates of stent thrombosis and restenosis, making PCI a viable alternative to saphenous vein grafts in select patients. The cost of the hybrid approach may be higher compared to those of PCI alone; however, this may be offset in the long term by the superior results of using the internal mammary artery, but the cost may be less compared to traditional CABG surgery due to a less invasive procedure with a shorter hospital length of stay [1, 4-9].

\section{KARGER}

E-Mail karger@karger.com

www.karger.com/crd
Konstantinos Dean Boudoulas, MD

The Ohio State University

473 W 12th Avenue, Suite 200

Columbus, OH 43210 (USA)

E-Mail kdboudoulas@ osumc.edu 
In the last issue of Cardiology, Saha et al. [10] discussed at length the advantages and limitations of hybrid coronary revascularization. In addition, outcomes related to standard CABG and PCI are compared to the hybrid approach. The authors suggested that CABG remains the gold standard in patients with multivessel coronary artery disease; however, due to advantages in medical science and medical technology, the hybrid approach may be attractive in certain individuals, especially the elderly [1, $10-12]$.

\section{Eligibility for CABG, PCI, or Hybrid Coronary Revascularization}

"Variability is the Law of Life" Sir William Osler

Coronary artery disease is a complex, long-lasting, and continuously evolving inflammatory process with various clinical manifestations ranging from asymptomatic to stable angina, acute coronary syndromes, heart failure, and sudden cardiac death. Due to the nature of the disease, many patients may live for several years and often decades with it. Since the clinical presentation of coronary artery disease varies among patients, different therapeutic modalities for each patient at different stages of the disease are often used. In a patient with an ST elevation myocardial infarction, PCI is the procedure of choice, while in a patient with left main coronary artery stenosis associated with multivessel coronary artery disease without other major comorbidities, CABG is the procedure of choice in order to achieve complete revascularization [1, 2]. Patients who are candidates for hybrid coronary revascularization include those with significant LAD stenosis suitable for left internal mammary artery anastomosis plus significant coronary artery stenosis in at least one other major coronary artery suitable for PCI. Obviously, this does not mean that all eligible patients should be treated using this approach.

In a recent multicenter study that involved 11 medical centers in the USA, in which The Ohio State University was a participant, 6,669 patients had a diagnostic coronary angiogram that was reviewed by both an interventional cardiologist and a cardiac surgeon. In this evaluation, a very high consensus (97\%) between the interventional cardiologists and the cardiac surgeons was found in regards to eligibility for a hybrid approach to revascularization. Of these patients, 2,954 (44\%) did not have significant coronary artery stenosis, while 3,715 (56\%) had significant coronary artery stenosis $(\geq 70 \%)$ in at least one major coronary artery; of those patients with significant coronary artery stenosis only $454(12 \%)$ were anatomically eligible, while 3,216 (88\%) were not anatomically eligible, for the hybrid approach [11]. Patients ineligible for the hybrid approach had single-vessel coronary artery stenosis, prior CABG where the left internal mammary artery anastomosis to LAD was already used, LAD not suitable for grafting, or nonsignificant LAD disease.

It should be mentioned that only those patients who are candidates for the hybrid approach would also qualify for traditional CABG ( $12 \%$ of the population with significant coronary artery disease) since, as a general rule, patients who are not suitable for a left internal mammary artery anastomosis to LAD usually are not referred for CABG. In contrast, a large proportion of patients with significant coronary artery disease are potentially candidates for PCI.

\section{Hybrid Coronary Revascularization}

Hybrid coronary revascularization can be performed at different times or at the same time. If a staged approach is used, left internal mammary artery anastomosis to the LAD is usually performed first $[10,11]$. This approach allows perfusion to a larger area of the myocardium (more than 50\%) earlier in the revascularization process and while the patient is not on dual antiplatelet therapy that is required after PCI. Further, if PCI follows the left internal mammary artery anastomosis to LAD, arteriography of the LAD anastomosis can be performed to confirm patency and any possible defects. The cardiopulmonary bypass time, aortic clamp time, blood loss (i.e., chest tube drainage and blood transfusions), intubation time, length of stay in the intensive care unit, and total length of stay in the hospital are less with hybrid coronary revascularization compared to standard CABG. Further, clamping of the aorta that may be associated with stroke, especially in the elderly, is avoided with the hybrid approach, as the left internal mammary artery is the only graft utilized [4, 6, 12-16]. Hybrid coronary revascularization can also be performed at the same time in the hybrid operating room (see later).

There are only a few studies with a small number of patients comparing traditional CABG or PCI to hybrid coronary revascularization $[3,4,11]$. A study funded by the National Institutes of Health is underway, where the outcomes of hybrid coronary revascularization (left internal mammary artery to the LAD plus PCI to the remaining arteries with significant disease) will be com-
Boudoulas/Marmagkiolis/Pitsis/ Boudoulas 


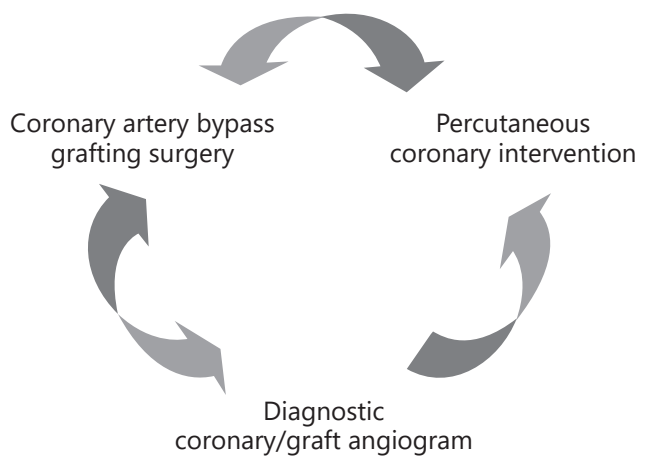

Fig. 1. In the hybrid cardiovascular operating room, a bypass graft angiogram is performed immediately after surgery and, if a corrective procedure is needed, surgery and/or percutaneous coronary intervention can be performed prior to chest closure.

pared to multivessel PCI (stents in all coronary arteries with significant stenosis including the LAD). We believe that an additional study comparing standard CABG to hybrid coronary revascularization should be conducted. The results of this and other studies may provide important information related to the optimal use of hybrid coronary revascularization. At present, it is our opinion that all patients who have an LAD stenosis suitable for internal mammary artery anastomosis and at least a significant coronary artery stenosis in another vessel suitable for PCI should be considered for the hybrid approach. In patients who are at a higher risk for traditional CABG, have multiple comorbidities, malignancy, and atherosclerosis of the aorta and/or aortic calcification who are at a higher risk for stroke during a standard CABG procedure may benefit the most from a hybrid approach. Current knowledge, sound clinical judgment, and common sense should be used to identify such patients.

\section{Hybrid Cardiovascular Operating Room}

"United we stand, divided we fall" Aesop, 620-564 BC

Performing hybrid coronary revascularization in the hybrid cardiovascular operating room allows the opportunity to perform an angiogram of the bypass graft immediately after surgery and before closure of the chest.
Using this approach in an earlier study from Vanderbilt University, it was found that the rate of overall graft defects following coronary artery bypass surgery requiring repair was $12 \%$, and the rate of defects of the left internal mammary to $\mathrm{LAD}$ requiring repair was $7 \%$. Thus, one of the major advantages of the hybrid cardiovascular operating room is that it allows an angiogram to be performed on all grafts (especially the left internal mammary artery to the LAD) and if necessary a corrective procedure (surgery and/or PCI) can be performed prior to chest closure [17] (Fig. 1). This approach will improve graft patency and long-term outcomes. Further, a hybrid approach in the hybrid cardiovascular operating room can be used for other cardiovascular procedures such as coronary artery revascularization plus valve replacement or repair, coronary artery revascularization plus aortic aneurysm repair, coronary plus peripheral artery revascularization (carotids and extremities), and any combination of the above, just to mention a few.

For these reasons, it is anticipated that in the near future hybrid cardiovascular operating rooms will be available in all major hospitals. Importantly, the hybrid cardiovascular operating room will bring all cardiovascular subspecialties (cardiologists, cardiac/vascular surgeons, cardiac anesthesiologists, and others) closer together. These close collaborations of the "heart team" will result in a better understanding of cardiovascular diseases, a more rational approach to the management of these diseases, and better outcomes.

\section{Conflict of Interest}

None.

References

$\checkmark 1$ Boudoulas KD, Triposkiadis F, Geleris P, Boudoulas H: Coronary atherosclerosis: pathophysiologic basis for diagnosis and management. Progr Cardiovasc Dis 2016;58: 676-692.

2 Head SJ, Milojevic M, Daemen J, et al: Mortality after coronary artery bypass grafting versus percutaneous coronary intervention with stenting for coronary artery disease: a pooled analysis of individual patient data. Lancet 2018;391:939-948.

-3 Harskamp RE, Brennan JM, Xian Y, et al: Practice patterns and clinical outcomes after hybrid coronary revascularization in the United States: a meta-analysis of $1,190 \mathrm{pa}-$ tients. Am Heart J 2014;167:585-592. 
4 Shen L, Hu S, Wang H, et al: One-stop hybrid coronary revascularization versus coronary artery bypass grafting and percutaneous coronary intervention for the treatment of multivessel coronary artery disease: 3 -year followup results from a single institution. J Am Coll Cardiol 2013;61:2525-2533.

5 Shroyer AL, Hattler B, Wagner TH, et al: Fiveyear outcomes after on-pump and off-pump coronary artery bypass. N Engl J Med 2017; 377:623-632.

-6 Leacche M, Byrne JG, Solenkova NS, et al: Comparison of 30-day outcomes of coronary artery bypass grafting surgery versus hybrid coronary revascularization stratified by SYNTAX and euroSCORE. J Thorac Cardiovasc Surg 2013;145:1004-1012.

7 Boudoulas KD, Triposkiadis F, Stefanadis C, Boudoulas $\mathrm{H}$ : The endlessness evolution of medicine, continuous increase in life expectancy and constant role of the physician. Hellenic J Cardiol 2017;58:322-330.
8 Boudoulas KD, Stefanadis C, Boudoulas H: The role of interventional cardiology to out understanding of basic mechanisms related to coronary atherosclerosis: "thinking outside the box." Hellenic J Cardiol 2017;58:58:110114.

-9 Yamaji K, Raber L, Zanchin T, et al: Ten-year clinical outcomes of first-generation drugeluting stents: the serolimus-eluting vs. paclitaxel-eluting stents for coronary revascularization (SIRTAX) VERY LATE trial. Eur Heart J 2016;37:3386-3395.

10 Saha T, Naqvi SY, Goldberg S: Hybrid revascularization: a review. Cardiology 2018;140: 35-44.

11 Puskas JD, Halkos ME, DeRose JJ, et al: Hybrid coronary revascularization for the treatment of multivessel coronary artery disease: a multicenter observational study. J Am Coll Cardiol 2016;68:356-365.

$12 \mathrm{Hu}$ S, Li Q, Gao P, et al: Simultaneous hybrid revascularization versus off-pump coronary artery bypass for multivessel coronary artery disease. Ann Thorac Surg 2011;91:432-438.

13 Reicher B, Poston RS, Mehra MR, et al: Simultaneous "hybrid" percutaneous coronary intervention and minimally invasive surgical bypass grafting: feasibility, safety, and clinical outcomes. Am Heart J 2008;155:661-667.
14 Bachinsky WB, Abdelsalam M, Boga, et al: Comparative study of same sitting hybrid coronary artery revascularization versus offpump coronary artery bypass in multivessel coronary artery disease. J Interv Cardiol 2012; 25:460-468.

15 Kon ZN, Brown EN, Tran R, et al: Simultaneous hybrid coronary revascularization reduces postoperative morbidity compared with results from conventional off-pump coronary artery bypass. J Thorac Cardiovasc Surg 2008; 135:367-375.

16 Zhou S, Fang Z, Xiong H, et al: Effect of onestop hybrid coronary revascularization on postoperative renal function and bleeding: a comparison study with off-pump coronary artery bypass grafting surgery. J Thorac Cardiovasc Surg 2014;147:1511-1516.

17 Zhao DX, Leacche M, Balaguer JH, Boudoulas KD, Damp JA, Greelish JP, et al: Routine intraoperative completion angiography after coronary artery bypass and 1-stop hybrid revascularization results from a fully integrated hybrid catheterization laboratory/operating room. J Am Coll Cardiol 2009;53:232-241. 